\title{
EL EMPOBRECIMIENTO DE LA INVESTIGACIÓN CUALITATIVA EN PSICOLOGÍA
}

\author{
THE IMPOVERISHMENT OF QUALITATIVE RESEARCH IN PSYCHOLOGY \\ ÓSCAR GILBERTO HERNÁNDEZ* \\ UNIVERSIDAD DE SAN BUENAVENTURA, BOGOTÁ - COLOMBIA
}

FECHA RECEPCIÓN: 21/1/2013・ FECHA ACEPTACIÓN: 21/02/2013

\begin{abstract}
Resumen
El texto presenta una reflexión sobre el sentido de la investigación cualitativa en psicología. Para ello se examina su definición tradicional, su relación con el conocimiento psicológico y la instrumentalización de sus técnicas. Se defiende la investigación cualitativa como una actividad científica, caracterizada por el rigor, la sistematicidad y la argumentación. Se proponen opciones para su auto fortalecimiento.

Palabras clave: metodología cualitativa, investigación, psicología.
\end{abstract}

\section{Abstract}

The text presents a reflection on the meaning of qualitative research in psychology. This examines its traditional definition, its relationship to psychological knowledge and the manipulation of their techniques. Qualitative research is advocated as a scientific activity, characterized by rigor, systematicity and argumentation. Options are proposed for self-empowerment.

Keywords: methodology, qualitative research, psychology.

\section{Método científico}

Definir la investigación cualitativa como aquella opuesta a la investigación cuantitativa es una falacia llena de peligros. Entre estos pueden contarse la subordinación de la primera a la segunda, la renuncia por consolidar su estatus científico y el abandono para impulsar su desarrollo independiente. Además, se trata de una definición circular que impide otras posibilidades. Es como si el color negro fuera la ausencia del color blanco -y viceversa-, o como si el frío fuera lo opuesto al calor. Tanto el negro como el frío son más que esto, así como la investigación cualitativa es mucho más que lo contrario a la investigación cuantitativa. Todas estas son definiciones simples.

La subordinación de la investigación cualitativa significa adoptar principios ontológicos provenientes de la investigación cuantitativa. Aunque parezca una combinación poco probable, su resultado es la investigación cualitativa positivista. Aquí, el término «positivista» no debe entenderse en forma despectiva. Este se refiere a un modo muy importante para sostener el conocimiento científico, con ventajas tan destacadas como lo son sus limitaciones. El problema fundamental surge cuando solo se reedifican sus ventajas y se ignoran sus restricciones.

* Doctorando de la Facultad de Psicología de la Universidad de Buenos Aires. Especialista en Políticas Educativas y Magíster en Ciencias Sociales con Mención en Educación, Facultad Latinoamericana de Ciencias Sociales -FLACSO- Sede Académica Argentina. Psicólogo, Universidad Nacional de Colombia. E-mail: oscararod@gmail.com 
La investigación cualitativa positivista se preocupa constantemente por cumplir los requisitos de cientificidad propios de la tradición cuantitativa, razón por la cual, se asemeja a un discípulo cuyo único propósito es parecerse a su maestro. Este será un discípulo condenado a la ceguera eterna.

Tal subordinación puede entenderse cuando solo se acepta un único modelo de ciencia. Como es sabido, el clásico «método científico», impulsado indirectamente por R. Descartes (1992) a mitad del siglo XVII en Europa, tuvo una difusión tan potente que se fue naturalizando en casi todo el planeta. La obsesión por la medición, el control y la predicción fue convirtiéndose en parte de la ciencia humana, a tal punto, que todo aquel que quisiera llamarse científico tendría que incorporar semejante trilogía. También es sabido que este método fue originalmente creado para las ciencias naturales y que su importación a las ciencias sociales presentó múltiples controversias. Pensar la investigación cualitativa como una actividad científica según el modelo descrito es una ilusión asombrosa. En vez de cultivar su método científico, debe cultivarse su proceso científico.

Si se elige tal opción, es decir, implementar el «método científico» para la investigación cualitativa, se afrontan dos caminos: abandonar la racionalidad científica o desarrollar su propia cientificidad. La evasión es el camino más fácil porque asegura ciertos mínimos de existencia y permite la adhesión a posturas livianas que renuncian al trabajo académico riguroso. En cambio, el segundo camino es más difícil porque implica una discusión epistemológica profunda, estudiando y pensando alternativas que en principio parecen imposibles. Por eso, varios investigadores cualitativos prefieren entender los saberes como disciplinas, y no como ciencias, como si su trabajo no fuera científico o como si el término «ciencia» fuese exclusivo de los investigadores cuantitativos. Al parecer, en la actualidad existe preferencia por el primer camino. El desarrollo científico de la investigación cualitativa aún depende de un solo modelo de ciencia.

\section{Psicología}

El sociólogo francés Bernard Lahire (2005) dice que los objetos de estudio no son en sí mismos psico- lógicos, antropológicos o sociológicos, sino que es la perspectiva teórica del investigador la que los determina. Y tiene razón porque los fenómenos no son exclusivos de ninguna ciencia sino que estas se los apropian y los fragmentan. En el caso de la psicología se reconocen sus enfrentamientos cuando trata de definirse: analizar el comportamiento, el inconsciente, la subjetividad humana, el cerebro, etc. Además puede decirse que las ciencias configuran -crean-, sus propios objetos de estudio con el propósito de ganar legitimidad y reconocimiento. Sin embargo, al momento de consolidar la forma en que ese conocimiento específico se construye pierden impulso y terminan atrapadas en una sola dimensión epistemológica.

La relación entre la investigación cualitativa y el conocimiento psicológico también es problemática. Desde luego, existen formas concretas de comprender la psicología que excluyen esa posibilidad y otras que la aceptan tímidamente. Es tímida porque comparada con otras ciencias sociales, la psicología muestra una incorporación modesta. No obstante, este es un evento significativo porque la investigación cualitativa en psicología cuestiona su propia tradición científica general. En su afán por adquirir un «verdadero»estatus científico, derivado de las ciencias naturales, la historia de la psicología podría constituirse en torno a este problema. Es decir, una historia atravesada por la preocupación metodológica. Por tales razones, cuando la investigación cualitativa irrumpe en la psicología abre posibilidades para pensar, incluso, otras alternativas de su historia.

Pero, ¿esto significa que la psicología está fragmentada? iNo!, lo que está fragmentado es el modo como esta construye su conocimiento, y a su vez, la forma como comprende su objeto de estudio. Así por ejemplo, se podría explorar la posibilidad de conectar los resultados de investigaciones en neuropsicología, sobre el proceso cerebral de atribución de significados, con los principios epistemológicos de la investigación cualitativa. Aunque parece una contradicción insuperable, puede notarse que ambos elementos están orientados en el mismo sentido: la interpretación. De igual manera, este razonamiento puede ser aplicado a otras opciones, siempre y cuando, conserve una misma línea ontológica. De lo contrario, es fácil adherirse al eclecticismo absurdo. 
Si pareciese que la psicología contemporánea está fragmentada es porque los psicólogos se han encargado de fragmentarla.

Entre los autores latinoamericanos que recientemente han enfrentado estas cuestiones sobresalen, el venezolano Miguel Martínez Miguélez (2006), curiosamente más conocido en el mundo de la ingeniería que en el de las ciencias sociales, y el cubano Fernando González Rey (2007), quien decididamente orienta su análisis a la psicología. Con algunas diferencias mínimas, ambos señalan que el problema de la relación entre la investigación cualitativa y la ciencia psicológica tiene un carácter epistemológico. Esto significa que para pensar seriamente en dicha relación debe acudirse al conocimiento epistemológico, so pena de incurrir en ilusiones procedimentales. Sin una referencia epistemológica elemental y sin ninguna connotación ontológica, también elemental, la investigación cualitativa en psicología resulta incomprensible. Por eso, para algunos investigadores, la investigación cualitativa se percibe de forma displicente. Es como si fuesen prisioneros de una matriz epistémica que no pueden observar.

\section{Proceso científico}

Debe recordarse que no existe contradicción entre la investigación cualitativa y las nociones de rigor académico, sistematicidad y argumentación. Aunque algunos investigadores las han abandonado injustificadamente, es indispensable revitalizarlas. En ocasiones, la defensa de estas nociones se confunde con la defensa de la epistemología positivista porque se cree que son de su propiedad exclusiva. Esta confusión puede concebirse como un prejuicio, en el sentido exacto de aquel definido por la psicología social:

No hay ningún prejuicio, e incluso tiene virtudes positivas, continuar usando la expresión ciencias sociales. Las ciencias sociales comparten con las ciencias naturales la claridad lógica en la formulación de teorías y una investigación sistemática. Pero la ciencia social no es un vapuleado y pesado barco de vapor que avanza infructuosamente tras la estela del ligero crucero de las ciencias naturales. En gran medida, las dos navegan en diferen- tes océanos, por mucho que compartan ciertos procedimientos de navegación comunes (Giddens, 1997: 108).

Esta analogía puede extrapolarse a la distinción entre la investigación cuantitativa y la cualitativa. Pese a sus notables diferencias, ambas comparten la claridad lógica y la sistematicidad, de tal manera, que las dos terminan siendo investigación científica. Si la investigación cualitativa enfatiza su adjetivo y olvida su sustantivo, puede padecer serias secuelas y estancarse.

Por ejemplo, la consecuencia reiterativa cuando se ignora el debate epistemológico sobre la investigación cualitativa en psicología es transferirlo a su aspecto metodológico. Es decir, comprender las técnicas de construcción de información como la instancia principal que la define. Desconocer la dimensión epistemológica de la investigación cualitativa en psicología implica instrumentalizar sus técnicas. Esto significa que el sentido fundamental de cada técnica se reduce a su procedimiento y se cristaliza, a tal punto, que se olvida el protagonismo intelectual del investigador y la connotación subjetiva de los participantes. De este modo, las entrevistas en profundidad carecen de profundidad, los grupos de discusión carecen de discusión, y los diarios de campo carecen de campo, entendido como el nivel de involucramiento con las comunidades. Instrumentalizar las técnicas de investigación cualitativa es rendir culto epistemológico al método, sacrificando la producción de ideas.

En ese sentido, valdría la pena pensar las implicaciones de reemplazar el término «método científico» por el de «proceso científico», para el caso de la investigación cualitativa en psicología. Más que una discusión lingüística, este cambio favorecería la consolidación de sus criterios de legitimidad, conectándolos con los avances de otros autores (Parker, 2004). Así se obtendría mayor desarrollo interno e independiente, porque la cuestión sobre el proceso científico sería transversal para quienes se dedican a esta actividad. Es como si un grupo de cultivadores se preocupara más por la forma en que siembran sus productos que por los productos en sí mismos. Con el tiempo descubrirán que la calidad de sus frutos depende de la calidad con la que los plantan. De igual manera, con el término «proceso científico», la investigación cualitativa en psicología recuperaría la discusión sobre la construcción de su conocimiento. 
Por tanto, si se ubica la legitimidad de la investigación cualitativa en la calidad de la expresión de los participantes, así como en la lógica de las interpretaciones de los investigadores, la idea de proceso ganaría más fuerza. Estos últimos tendrían que comunicar sus detalles, mostrando los raciocinios en la elección de instrumentos y en la configuración de los resultados. Además, esta perspectiva permitiría superar la instrumentalización de técnicas mencionada, porque privilegia la consistencia del razonamiento sobre la consistencia del procedimiento. En últimas, no se trata solo de aprender a desarrollar las operaciones propias de la investigación cualitativa en psicología, sino además, de aprender a reflexionar sobre lo que en ellas acontece. Es distinto ser técnico de investigación que profesional de investigación.

\section{Síntesis}

El empobrecimiento de la investigación cualitativa en psicología consiste en adaptar (directa e indirectamente) la epistemología positivista proveniente de su tradición cuantitativa. También consiste en instrumentalizar sus técnicas, renunciando a la producción de ideas por rendir culto a su método. Como resultado, su rigor académico, sistematicidad y argumentación se debilitan creyendo que estas son propiedades exclusivas de la investigación cuantitativa. La responsabilidad de este empobrecimiento no está en los críticos de la investigación cualitativa, sino en los mismos investigadores cualitativos, quienes poco a poco abandonan el carácter científico de su trabajo. Una alternativa interesante es redimir su cualidad epistemológica y teórica subyacente para proponer opciones más complejas. Después de todo, hay que tener en cuenta que «los datos solo logran hablar cuando la teoría les aporta el don del lenguaje» (Boron, 2010: 14).

\section{Referencias}

Boron, A. (2010). Introducción. En Sautu, R (Comp.). Manual de metodología. Buenos Aires: Consejo Latinoamericano de Ciencias Sociales - Prometeo.

Descartes, R. [1637] (1992). El discurso del método. Traducción, cronología, bibliografía y notas de Jorge Aurelio Díaz. Bogotá: Norma.

Giddens, A. (1997). Sociología. Madrid: Alianza.

González, F. (2007). Investigación cualitativa y subjetividad. Los procesos de construcción de la información. México: McGraw-Hill.

Lahire, B. (2005). De la teoría del habitus a la sociología psicológica. En B. Lahire (Comp.). El trabajo sociológico de Pierre Bourdieu: Deudas y críticas. Buenos Aires: Siglo XXI.

Martínez-Miguélez, M. (2006). Ciencia y arte en la investigación cualitativa. México: Trillas.

Parker, I. (2004). Criteria for Qualitative Research in Psychology. Qualitative Research in Psychology, 1, 1-12. 